\title{
148. On the Ring 13 Chromosome in a Malformed Infant with Special Regard to the Break Point
}

\author{
By Yoshikazu KurokI*) and Yuko Nagano**) \\ (Comm. by Sajiro Makino, M. J. A., Oct. 12, 1974)
}

This paper reports preliminarily the ring $\mathrm{D}$ chromosome in a one-year-old male infant having multiple malformations. The aberrant chromosome was analysed by the Giemsa banding technique, with particular concern to break points. No correlation between the development of retinoblastoma and the specific deleted band in the long arm of No. 13 chromosome was noted in this patient.

Case report. A one-year-old boy was the product of an uneventful course of pregnancy and normal delivery. The patient's mother and father were 21 and 23 years old at his birth and showed no physical anomaly. The family history showed no special disorders. The patient weighed $1770 \mathrm{~g}$, measured $43 \mathrm{~cm}$ in length, and $30.1 \mathrm{~cm}$ in head circumferance at birth. Inperforate anus and malrotation of the intestine were noted in his neonatal period. The patient received surgical operations. The postoperative course was uneventful, but somatic growth failure and mental retardation were remarkable.

His main clinical features were physical and mental retardation, somatic growth failure, microcephaly with flat occiput, large earlobes, protruding maxilla, micrognathia and short neck. The postoperative scars of anal atresia and intestinal malrotation were noted. The external genitalia were abnormal showing bilateral cryptorchidism and hypoplastic scrotum. Retinoblastoma was not recognized in this patient. Dermatoglyphics was within normal limits, except for absent $b$ triradius.

Routine laboratory examinations, including hematological examinations, urinalysis, and blood chemistry, were all within normal limits. Hemoglobin-F was $6 \%$, indicating a moderate elevation. The patient's haptoglobin was type $\mathrm{Hp2-1}$, while it was also $\mathrm{Hp2-1}$ in both parents. Thus, the haptoglobin locus seemed not to be deleted in this case. An intravenous pyelography failed to demonstrate the right kidney.

\footnotetext{
*) National Fukuoka Central Hospital, Fukuoka.

**) Department of Pediatrics, Faculty of Medicine, Kyushu University, Fukuoka.

Request reprints from: Dr. Y. Kuroki, Division of Medical Genetics, Kanagawa Children's Medical Center, Yokohama, Japan, 233. (Present Address)
} 
[Vol. 50,
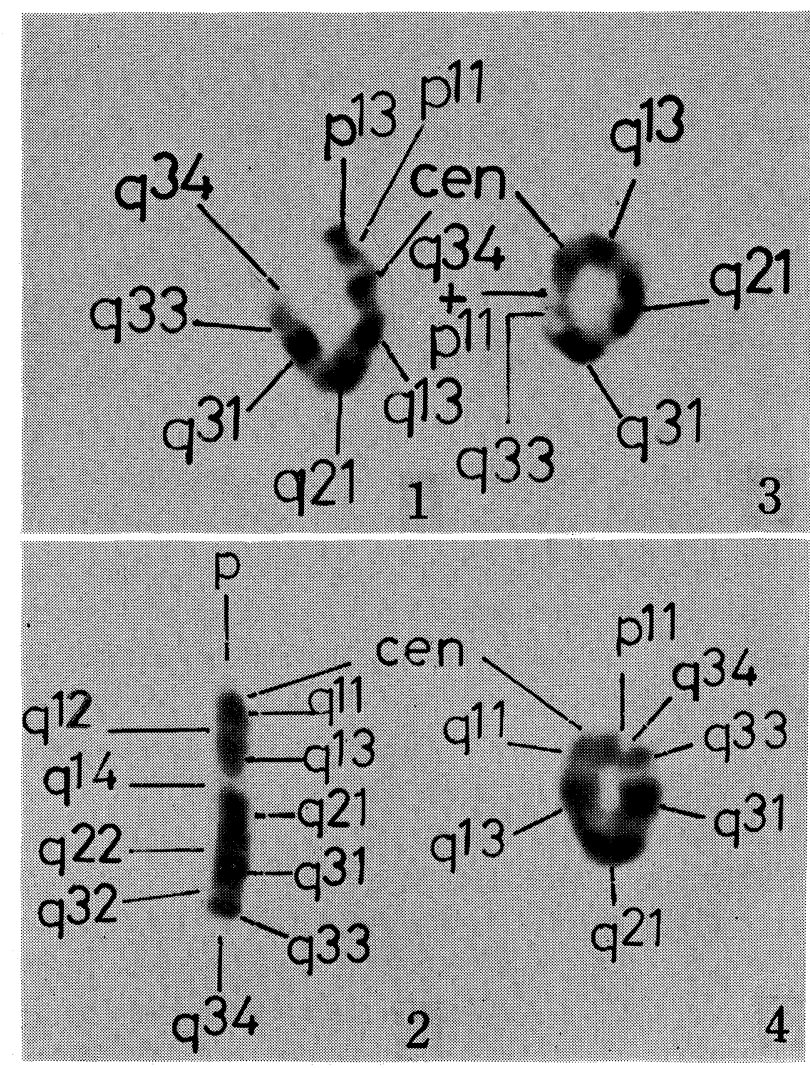

Figs. 1-4. Trypsin-Giemsa banding patterns of normal (1-2) and ring 13 chromosomes (3-4). All 4 dark bands in the long arm of the normal 13 chromosomes (1-2) can be recognized in the rings (3-4). The break points are located at bands $13 \mathrm{p} 11$ and $13 \mathrm{q} 34$ (3-4).

Cytogenetic findings. Peripheral lymphocytes of the patient cultured by the ordinary 3 -day-method indicated that in 40 cells examined, 31 cells $(77.5 \%)$ were found to be $46, \mathrm{XY}, \mathrm{r}(\mathrm{D})$, and 9 cells $(22.5 \%)$ were $45, \mathrm{XY},-\mathrm{D}$. By the 2 -day-method, 39 cells $(78 \%)$ were recognized as $46, \mathrm{XY}, \mathrm{r}(\mathrm{D})$ and 11 cells (22\%) as 45, XY,-D.

The trypsin-Giemsa banding technique demonstrated the ring $\mathrm{D}$ chromosome as a ring 13 . The break points were found to be located at bands 13p11 and 13q34. All four dark bands in the long arm of chromosome 13 were noticed. Thus, the loss of chromosome material was estimated to be very small.

Remarks. Clinical features of this case was consistent with the ring 13 syndrome so far reported. Since the chromosomal loss in the r(13) syndrome occurs in the long arm of the No. 13 chromosome, the 13q- syndrome and the $\mathrm{r}(13)$ syndrome share similar clinical fea- 
tures. The clinical pictures characteristic of the two syndromes are mental retardation, microcephaly, trigonocephaly, large earlobes, protruding maxilla (or incisor), micrognathia and undescended testis. Inperforate anus and the abnormal inheritance of haptoglobin are frequently noticed in the $\mathrm{r}(13)$ cases.

Recently, Nakagome et al. (1973) investigated the break points in a ring 5 cat-cry-syndrome patient by the G-banding technique. In our $r(13)$ case, break points were found to be located at 13p11 and 13q34 bands, resulting in a small amount of the chromosomal loss.

Orye et al. (1971) reported a case of the 13q- syndrome with retinoblastoma and showed that the missing portion of No. 13 chromosome was around the broadest band on its long arm. Willson et al. (1973) described a 13q- patient with retinoblastoma with the identification of the deleted band as 13q31.

The deleted portions of our case seemed to be some parts of $13 \mathrm{p} 11$ and 13q34, retaining the band 13q22 intact. It is commented that the present patient has no retinoblastoma at the age of one year, and may be free from developing the tumor in the future.

Acknowledgements. We are grateful to Professor Emeritus Dr. Sajiro Makino, and Professor Dr. Motomichi Sasaki, Hokkaido University, for reading the manuscript.

\section{References}

Nakagome, Y., Iinuma, K., and Matsui, I. (1973) : A trisomy 10 with mosaicism. A clinical and cytogenetic entity. Jap. J. Hum. Genet., 18, 216-219.

Orye, E., Delbeke, M. J., and Vandenabeele, B. (1971): Retinoblastoma and D chromosome deletions. Lancet, ii, 1376.

Willson, M. G., Towner, J. W., and Fujimoto, A. (1973) : Retinoblastoma and D chromosome deletions. Amer. J. Human Genet., 25, 57-61. 